\title{
Committees and Acknowledgements to the reviewers
}

\section{Organising committee}

- Pierre Delage (Conference chair)

- Yu-Jun Cui (Conference co-chair)

- Siavash Ghabezloo

- Jean-Michel Pereira

- Anh-Minh Tang

- Séverine Beaunier

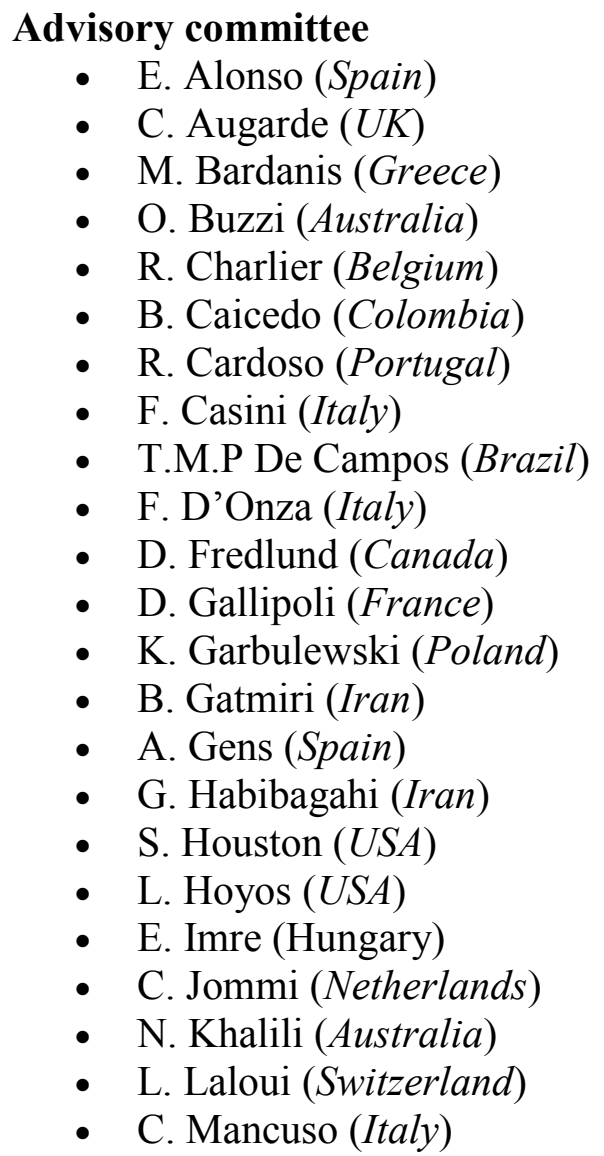

- D. Manzanal (Argentina)

- F. Marinho (Brazil)

- D. Masin (Czech Republic)

- F. Masrouri (France)

- G. Miller (USA)

- C. Ng (Hong Kong)

- T. Nishimura (Japan)

- L. Oldecop (Argentina)

- H. Rahardjo (Singapore)

- E. Romero (Spain)

- A. Russell (Australia)

- M. Sanchez (USA)

- T. Schanz (Germany)

- D. Sheng (Australia)

- S. Springman (Switzerland)

- H.W. Sun (Hong Kong)

- D. Toll (UK)

- S. Tripathy $(U K)$

- S. Vanapalli (Canada)

- J. Vaunat (Spain)

- S. Wheeler $(U K)$

- T. Zhang (China)

Careful reviewing is an essential process to ensure the high quality of the papers accepted at the Conference and published in the Proceedings. The Organising Committee is most grateful to the following reviewers for their kind and helpful contribution to the success of the Conference.

Alonso Eduardo, Ashayeri Iman, Augarde Charles, Baille Wiebke, Bicalho Katia, Biglari Mahnoosh, Buzzi Olivier, Caicedo Bernardo, Cardoso Rafaela, Chen Yonggui, Cordão-Neto Manoel, Cuisinier Olivier, Dangla Patrick, Deng Yongfeng, Fabbri Antonin, Feia Sadok, Fleureau Jean-Marie, Fredlund Delwyn, Gallipoli Domenico, Gens Antonio, Ghayoomi Majid, Habibagahi Ghassem, Heyerdahl Håkon, Hong Zhenshun, Khelifa Abbeche, Le Pense Solenn, Lloret Antonio, Manzanal Diego, Mašin David, Mendes João, Morvan Mathilde, Nikooee Ehsan, Nishimura Tomoyoshi, Rahardjo Harianto, Roux Jean-Noël, Russo Giacomo, Salager Simon, Seyedi Darius, Sheng Daichao, Stefanou Ioannis, Sun Wenjing, Tang Chao-Sheng, Teng Jidong, Tripathy Snehasis, Vanapalli Sai, Villar María Victoria, Wang Qiong, Zeng Ling-Ling, Zha Fusheng, Zhou Annan. 